Academic Platform Journal of Engineering and Science

journal homepage: http://apjes.com/

\title{
Bound Water and Hydroxyproline are the essential contributors to collagen molecular stability: A Computational Analysis
}

\author{
*Mustafa Ünal \\ ${ }^{1}$ Karamanoglu Mehmetbey University, Karaman, Turkey \\ mustafaunal@kmu.edu.tr, (D)
}

Research Paper

Arrival Date: 20.01.2019

Accepted Date: 23.06.2019

\begin{abstract}
Being the primarily organic phase of bone, collagen type I is an important contributor to bone's mechanical resistance to fracture. Gaining mechanistic insight into collagen stabilization mechanism is critical to developing new targets to prevent bone fracture. The role of water and hydroxyproline (Hyp) in collagen stability mechanism is still controversial. The aim of this study was to investigate the influences of Hyp and bound water on the collagen molecular stability. Four collagen like-peptide (CLP) models were compared in terms of conformational energies and hydrogen bonding types. CLP1 model represents regular collagen structure without water molecules while CLP2 model represents collagen structure without water and Hyp residue. CLPW1 and CLPW2 are the models of CLP1 and CLP2 with water molecules around them, respectively. Cumulative interpreting of four CLPs models was shed light on the factors influencing collagen stability in the frame of steric energy. Total steric energy was ordered as: CLP2 > CLP1 > CLPW2 > CLPW1, indicating that CLPW1 was the most stable collagen model. On the other hand, CLP2 was the least stable collagen model based on the steric energy comparison. In addition, the hydrogen bonding observed in the four models reveled that water molecules around the models help in binding collagen triple helix through different water bridges since they contributed extra way for binding of triple chains. Moreover, some of the observed water bridges involved directly the presence of Hyp residue. Cumulative results suggested the important role of bound water molecules and Hyp on collagen molecular stability.
\end{abstract}

Keywords: Collagen, Bound Water, Hydroxyproline, Computational Molecular Mechanics, Bone

\section{INTRODUCTION}

Bone is a complex natural material comprised of mineral, organic matrix and water [1]. Type I collagen ( $~ 90 \%$ of total organic matrix) in a hydrated environment is the main composition of bone's organic phase. Other components of organic matrix include non-collagenous proteins such as osteopontin, osteocalcin, and fibrillin [1, 2]. Bone's resistance to fracture originates from both its unique composition and unique arrangement of the primary constituents [1]. While mineral phase of bone primarily contributes to strength and stiffness of bone, interlinked collagen matrix in hydrated environment confers bone's ductility (toughness) and post-yield deformation [1, 3]. Collagen molecules' structural integrity is one of the main contributors to the structural integrity of bone's organic matrix that consequently influences mechanical behavioral of bone [4].

The current concept of collagen molecule is triple polypeptide helices composed of two identical $\alpha-1$ chains and one slightly different $\alpha-2$ chain (each chain of collagen molecule includes more than 1000 amino acid residues) [57].The supercoiled right-handed triple helix (tropocollagen) is almost $300 \mathrm{~nm}$ in length and $1.6 \mathrm{~nm}$ in diameter [5, 6]. The most common amino acid sequence of collagen molecule is the multiple repeated of (Glycine (Gly)-X-Y) triplet, where $\mathrm{X}$ and $\mathrm{Y}$ refer mostly to Proline (Pro) and Hydroxyproline (Hyp), respectively [8, 9] (Figure 1). Hyp is the result of post-transitional enzymatic modification of Pro, and always finds in collagen molecules in bone.

The structure of collagen molecule and its stability mechanism have been studied for the decades. In 1954, the first collagen model was developed by Ramachandran and Kartha [10] and this model was later refined by Rick and Crick in 1961 [6]. In 1980, the first crystal structure of collagen was obtained as (Pro-Pro-Gly) $)_{n}$ [11]. The first high resolution of collagen-like peptide (CLP) was reported in 1994 [12] and this structure confirmed to the existence model of Ramachandran and Kartha in 1954 [10]. Afterwards, many studies on collagen triple helix have been performed to characterize tropocollagen structural properties, collagen stability as well as folding mechanism [7]. 


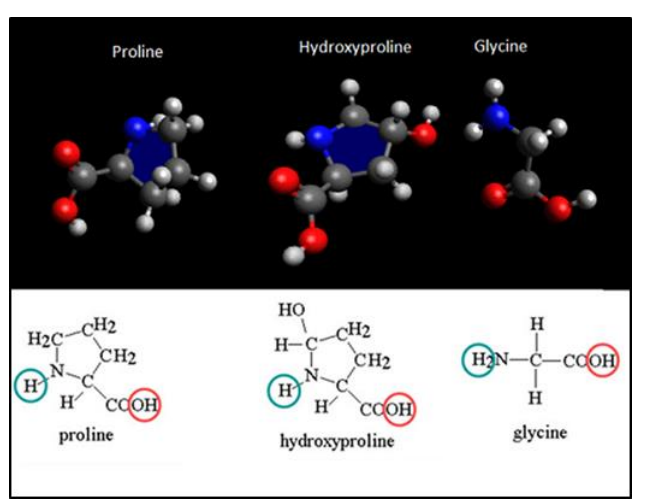

Figure 1. Individual amino acid types which were used in this study for modeling CLP and CLPW models. Blue and red circles indicate bonding site of amino acids.

In the literature, both experimental (i.e., thermodynamics experiment, X-ray crystallography or NMR) and computational molecular modeling research (i. e; molecular modeling simulation) have been directed to gain mechanistic insight to collagen stabilization mechanism [7]. However there is still no consensus of the role of Hyp and water on collagen stability/integrity. Some previous studies indicated that the presence of Hyp residue and water molecules have a major role in stabilization of collagen molecule through water bridges (interstitial water). These water bridges are connecting between amide and carbonyl groups at the site of substation [7, 12-20]. On the other hand, other studies (based on mainly experimental analysis) concluded that Hyp or/and water bridges cannot have a fundamental importance of collagen stability because when comparing (Pro-Hyp-Gly)n with (Pro-fPro-Gly) $)_{n}$ types of collagen-like peptide indicated that (Pro-fPro-Gly) $)_{n}$ was much more stable than (Pro-HypGly) $)_{n}$ even though hydrogen bonding ability of fPro (fluoroproline) is very weak [21-26]. Holmgren et al. [22, 23 ] suggested that the hydrogen bonding is not necessary to play an important role for stabilization of collagen. Furthermore, Engel et al [27] reported that (Pro-Hyp-Gly)n was more stable than (Pro-Pro-Gly) $)_{n}$ even in non-aqueous solution, suggesting that stability role of Hyp is not related to water bridges. Therefore, collagen stability mechanism has been still controversial, especially for the role of Hyp and water on collagen stability.

The aim of the study was to investigate the influences of Hyp and bound water architecture on the molecular stability of collagen using a computational molecular mechanics.

\section{METHODS}

\subsection{Construction of the molecular models}

Since atomic level analysis of full-length collagen has been difficult due to size of collagen and its fibrous structure, the collagen model with small number of residues (20-30 amino acid residues in the different form of (Gly-X-Y)) has been generally used for computational molecular modeling. This type model has been called as collagen-like peptide (CLP)
[12]. The initial CLP model was obtained from the RCSB Protein Data Bank (PDB) (PDB ID: 1CAG) [12], and all models were created by modifying this original CLP model such as replacing Hyp with Pro for CLP2 model or adding water molecules around it. In this study, the CLP model refers to collagen-like peptide without hydration environment while CLPW refers to collagen-like peptide with hydration environment. CLP1 model included (GlyPro-Hyp) ${ }_{10}$ peptide repeats, representing the regular collagen structure. CLP2 model is the repeat of (Gly-Pro-Pro $)_{10}$ sequence with Hyp residue absent (Figure 2). The coordinates of an idealized triple helix for process of coiling chains were adapted from the literature $[12,28]$. To create hydration environment around the CLP models, approximately 130 water molecules were firstly created by Avogadro software and same water construction was added with suitable distance around CLPW1 and CLPW2 models (Figure 3).

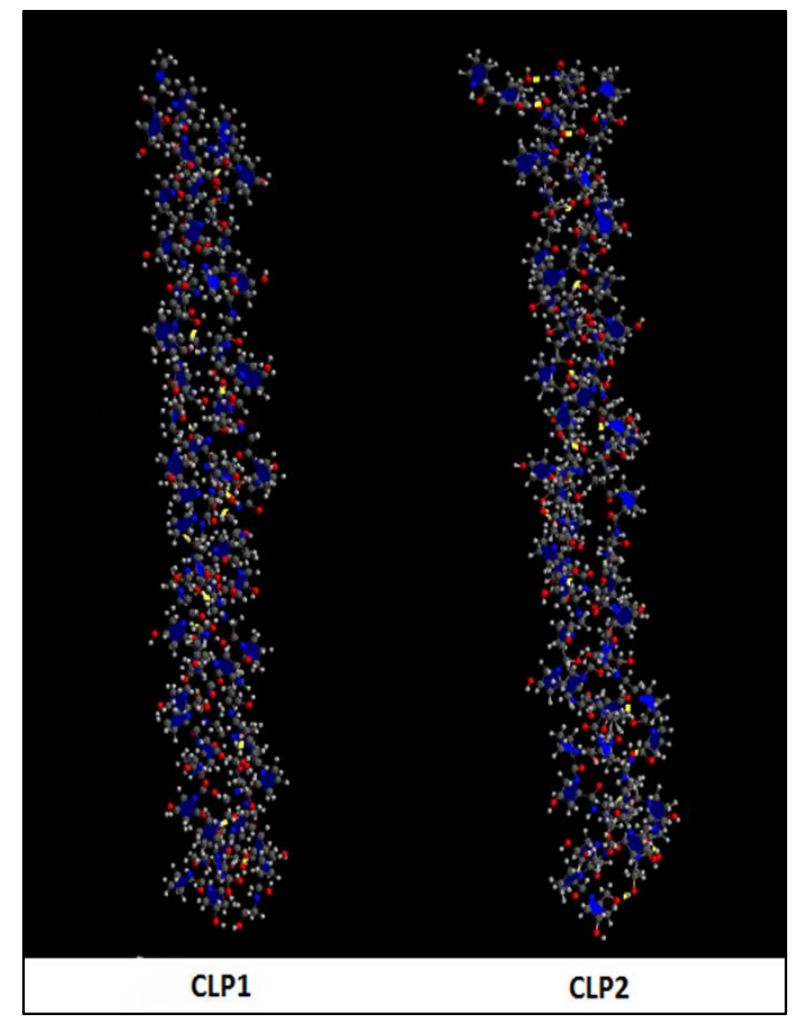

Figure 2. CLP1 and CLP2 models, representing collagenlike peptide without water molecules, after energy minimization process. Yellow shapes indicate the existence of hydrogen bonding sites.

\subsection{Software and parameters}

Molecular mechanics (MM) analyses were performed by using both Avogadro software with Merck Molecular Force Field (MMFF94)[29] and ChemBio3D Ultra version 12.0 with Molecular Mechanic force field (MM2) [30]. The main difference between MM2 and MMFF94 is that MMF94 has generally more terms in the force field such as more cubic terms in angle bending potential energy and more cubic and 
quartic terms in the bond stretch [31]. MMF94 is mostly used for protein analysis (see details in [29]). Since both force fields have some well-known advantages and disadvantages, using both of them provided better approach for energy minimization process.

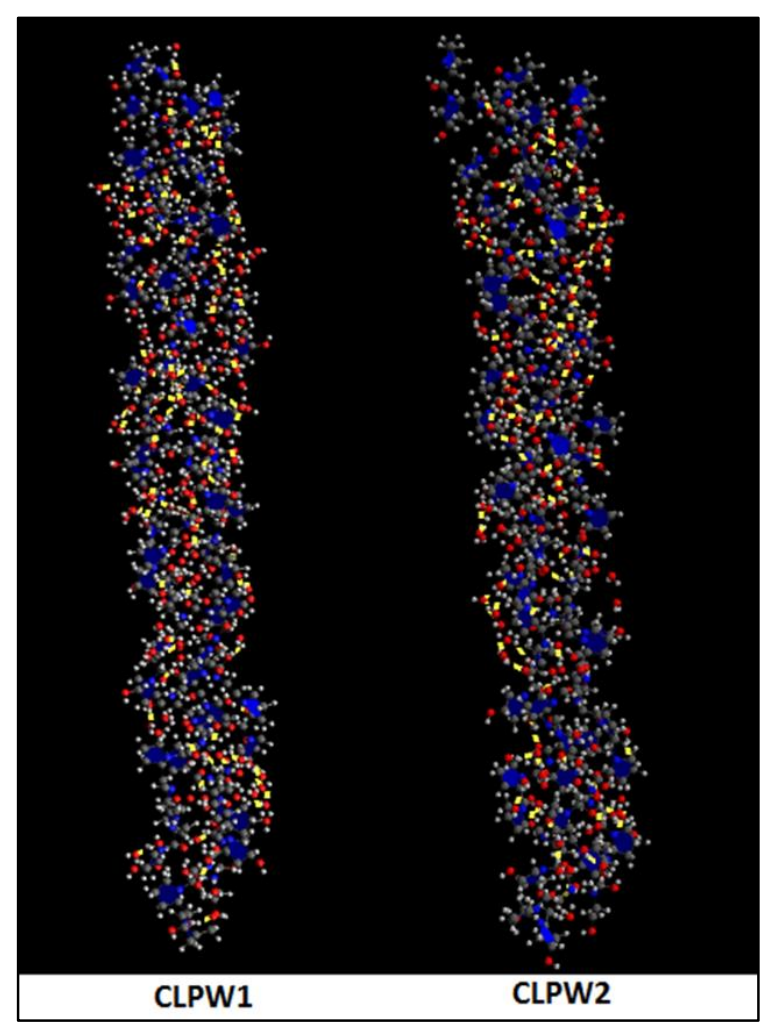

Figure 3. After energy minimization process, CLPW1 and CLPW2 models, representing collagen-like peptide with water molecules. Yellow shapes indicate existence of hydrogen bonding sites.

\subsection{Computational Analysis}

The stability collagen was analyzed in the perspective of steric (conformational) energy concept [31]. Steric energy is the energy due to geometry and molecule conformation, and is an important concept of computational biochemistry, especially for large molecules like proteins. Steric energy includes energy due to the bending, bond stretching, stretchbend, out of plane, and torsion interactions as well as electrostatic interaction and Van der walls interaction (see details in [31]). In the steric energy calculation, conformation of a molecule has the lowest energy conformation level because energy also wants to minimize itself in nature [31]. The lowest energy conformation, which is called energy minimization process, is set of bonded and angles, providing the smallest steric energy, and is inversely related with collagen stability: lower the steric energy, higher the collagen stability. In order to eliminate geometric strain and close contact in the CLP models and find steric (conformational) energy, resulting of stability of CLP model conformation, energy minimization process was performed with the following scheme: first, the CLP and CLPW models were minimized for 50 steps by steepest decent (SD) method with force field MMFF94 and 10e-5 convergence value. These parameters were chosen with considering computer limitations and avoiding time consumption (see details in $[31,32]$ for choosing parameter). Each energy minimization process of CLP models took 30 hours and process of CLPW models took slightly more than 30 hours. After first step completed, the processed models were minimized for a second time by MM2 force field with 0.1 RMS (root mean square) gradients. After energy minimization process was done, the model conformation was verified by comparing reported main chain conformational angles (dihedral angles) with the reported values in literature. Structural stability of CLP and CLPW models were analyzed by comparing the steric energies and by investigating the number and observed type of hydrogen bonds in the models.

\section{RESULTS}

Visual inspection of the models showed that structure of two models (CLP1 and CLP2) generally coincided with original tropocollagen conformation. CLP1 and CLP2 models were generally uniform and models were able to protect their integrity between triple helix ends (Figure 2 and 3). The structural conformation was confirmed through comparing mean torsion (dihedral) angles, $\phi$ and $\psi$, of the two models obtained from previously published works. The average $\phi$ angle was between $-67.78^{\circ}$ and $-72.90^{\circ}$ and the average $\psi$ angle was between $160.12^{\circ}$ and $164.80^{\circ}$ for CLP1. For CLP2, these values were between $-71.67^{\circ}$ and $-83.03^{\circ}$ and between $162.40^{\circ}$ and $167.18^{\circ}, \phi$ and $\psi$ respectively. Table 1 summarized comparison of the torsion angles of CLP models with available data from literature. Especially, $\psi$ angle value of Y position differed by up to $20^{\circ}$ between the CLP models and the values reported in the literature although rest of values coincided, depending on type of structure, with one or more data obtained from the literature.

Table 2 and 3 summarized the calculated conformational (steric) energies. Total steric energy of CLP1 was 683.7994 $\mathrm{kcal} / \mathrm{mol}$ while it was $-903.6372 \mathrm{kcal} / \mathrm{mol}$ for CLPW1 (Table 2 ). The main reason of considerable difference between steric energy of CLP1 and CLPW1 was related to values of total van der Walls (VDW) and Dipole/Dipole interaction energy. The absolute value of total van der Walls energy (Non-1,4 VDW and 1,4 VDW) increased from 220.6782 to $897.6418 \mathrm{kcal} / \mathrm{mol}$ while the absolute value of Dipole/Dipole interaction energy increased from 148.2414 to 1117.2804 $\mathrm{kcal} / \mathrm{mol}$ and the difference was $969.039 \mathrm{kcal} / \mathrm{mol}$. However, the bend, stretch and torsion energy were comparable between CLPW1 and CLP1 (Table 2). 
Table 1: Comparison of average main chain conformational (torsion) angles of CLP1 and CLP2 models with the angles of the previously published models.

\begin{tabular}{|l|l|l|l|l|l|l|l|l|}
\hline \multirow{3}{*}{ Angles } & $\begin{array}{l}\text { Residue } \\
\text { Position }\end{array}$ & CLP1 & CLP2 & $\begin{array}{l}\text { Crystal } \\
\text { structure of } \\
\text { collagen[33] }\end{array}$ & $\begin{array}{l}\text { (XYG) } \\
{[28]}\end{array}$ & $\begin{array}{l}\text { Collagen } \\
10_{7} \\
\text { Helix[34] }\end{array}$ & $\begin{array}{l}\text { (PPG) })_{10} 7_{5} \\
\text { Helix[11] }\end{array}$ & $\begin{array}{l}\text { Collagen- } \\
\text { like } \\
\text { peptide }\end{array}$ \\
\hline \multirow{2}{*}{$\phi\left(^{\circ}\right)$} & $\mathrm{X}$ & -72.90 & -71.67 & -71 & -87 & -72.1 & -75.5 & -72.60 \\
\cline { 2 - 10 } & $\mathrm{Y}$ & -72.18 & -73.79 & -66 & -69 & -75.0 & -62.6 & -59.60 \\
\cline { 2 - 10 } & Gly & -67.78 & -83.03 & -68 & -81 & -67.6 & -70.2 & -71.90 \\
\hline \multirow{2}{*}{$\psi\left(^{\circ}\right)$} & $\mathrm{X}$ & 161.32 & 163.57 & 160 & 155 & 164.3 & 152 & 163.8 \\
\cline { 2 - 9 } & $\mathrm{Y}$ & 160.12 & 167.18 & 148 & 146 & 155.8 & 147.2 & 149.8 \\
\cline { 2 - 9 } & Gly & 164.80 & 162.40 & 167 & 173 & 151.4 & 175.4 & 174.1 \\
\hline
\end{tabular}

Table 2: Steric energy values of CLP1 and CLPW1 after energy minimization/geometry optimization process.

\begin{tabular}{|c|c|c|}
\hline $\begin{array}{c}\text { Conformational (Steric) Energy } \\
(\mathrm{kcal} / \mathrm{mol})\end{array}$ & CLP1 & CLPW1 \\
\hline Stretch & 47.0139 & 47.3994 \\
\hline Bend & 366.1212 & 13.4466 \\
\hline Stretch-Bend & 12.8326 & 636.0825 \\
\hline Torsion & 626.7514 & -1303.3514 \\
\hline Non-1,4 VDW & -630.6017 & 405.7097 \\
\hline 1,4 VDW & 409.9235 & -1117.2804 \\
\hline Dipole/Dipole & -148.2414 & $-903.6372 \mathrm{kcal} / \mathrm{mol}$ \\
\hline Total Energy & $683.7994 \mathrm{kcal} / \mathrm{mol}$ & \\
\hline
\end{tabular}

Table 3: Steric energy computation of CLP2 and CLPW2 after energy minimization/geometry optimization process.

\begin{tabular}{|c|c|c|}
\hline $\begin{array}{c}\text { Conformational(Steric) Energy } \\
(\mathrm{kcal} / \mathrm{mol})\end{array}$ & CLP2 & CLPW2 \\
\hline Stretch & 47.9900 & 45.9011 \\
\hline Bend & 383.9867 & 401.3347 \\
\hline Stretch-Bend & 13.7077 & 641.8941 \\
\hline Torsion & 626.8381 & -1256.2341 \\
\hline Non-1,4 VDW & -614.7183 & 415.2250 \\
\hline 1,4 VDW & 407.0030 & -1114.0146 \\
\hline Dipole/Dipole & -137.3287 & $-853.1001 \mathrm{kcal} / \mathrm{mol}$ \\
\hline Total Energy & $727.4786 \mathrm{kcal} / \mathrm{mol}$ & \\
\hline
\end{tabular}

Total steric energy of CLP2 was $727.4786 \mathrm{kcal} / \mathrm{mol}$ while total steric energy of CLPW2 was $-853.1001 \mathrm{kcal} / \mathrm{mol}$ (Table $3)$. The difference between the steric energies was again related to VDW and Dipole/Dipole interaction energies. The total steric energies can be ordered as follows: CLP2 > CLP1 $>$ CLPW2 > CLPW1.

Hydrogen bond numbers with cut-off radius 2.00 angstroms (A) and cut-off angle $120^{\circ}$ were calculated by visual inspection. Table 4 summarized the observed hydrogen bond number of the models. Both CLW1 and CLPW2 had highest number of hydrogen bonds than CLP1 and CLP2 models have.

Several different types of hydrogen bonding were observed with respect to water and type of models (Figure 4-8). The most common interchain hydrogen bonding in the CLP models was $\mathrm{NH}_{(\mathrm{Gly})} \cdots \mathrm{O}=\mathrm{C}_{(\mathrm{Pro})}$ and it existed without hydration environment (Figure 5).
CLPW models had mostly two kinds of hydrogen bonding by contribution of water bridges: $\mathrm{N}-\mathrm{H}_{\text {(Gly or Pro) }} \cdots$ (Water $)_{n} \cdots$ $\mathrm{O}-\mathrm{H}_{(\mathrm{Hyp})}$ and $\mathrm{O}-\mathrm{H}_{(\mathrm{Hyp})} \cdots$ (Water) $)_{n} \cdots \mathrm{O}=\mathrm{C}_{(\mathrm{Gly}}$ or Pro) and both were observed as intrachain and interchain hydrogen bonding (Figure 6 and 7). $\mathrm{C}=\mathrm{O}_{\text {(any residue) }} \cdots$ (Water) $)_{\mathrm{n}} \cdots$ $\mathrm{O}=\mathrm{C}_{\text {(any residue) }}$ and $\mathrm{N}-\mathrm{H}_{\text {(any residue) }} \cdots$ (Water) $)_{\mathrm{n}} \cdots \mathrm{O}=\mathrm{C}_{\text {(any residue) }}$ were other types of intrachain and interchain hydrogen bonding (Figure 8).

Table 4: Total existed hydrogen bond numbers (cut-off radius: 2.00 angstroms and cut-off angle: $120^{\circ}$ ) of the models

\begin{tabular}{|c|c|}
\hline Models & $\begin{array}{c}\text { Existed Hydrogen Bond } \\
\text { numbers }\end{array}$ \\
\hline CLP1 & 20 \\
\hline CLPW1 & 128 \\
\hline CLP2 & 19 \\
\hline CLPW2 & 129 \\
\hline
\end{tabular}




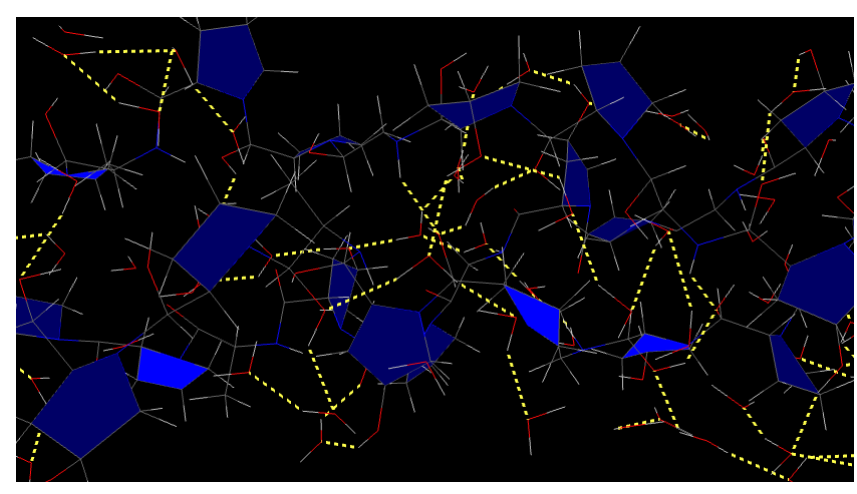

Figure 4. Overall view of several types of hydrogen bonding between the residues and water molecules in CLPW models. Yellow dot lines show the hydrogen bonds.

\section{DISCUSSION}

In the present study, four CLP models were analyzed to gain mechanistic insight to the effects of Hyp and bound water on collagen stability. The structural conformation of these models were confirmed by comparing dihedral (torsion) angles, $\phi$ and $\psi$ with obtained data from the literature [11, 12, 28, 33, 34]. The models have slightly different angle values from some of the previously published studies [10, 11, 26-28]. This difference could be related to using data from crystallographic experiments and geometry optimization process of the models. Nevertheless, each torsion angle coincides with one or more angles from the previous studies (Table 1). This result supports that overall structure of models is sufficiently suitable to analyze the stability of collagen.

The present study showed that CLPW1 has lesser steric energy compared to CLP1 (Table 2). Moreover, the steric energy value of CLPW2 has lower than that of CLP2 (Table 3). Previously, Tamilselvan and Zhang also showed that the value of steric energy of CLP with hydrated environment has lesser than that of the value without hydrated environment for several models obtained from RSCB Protein Data Bank [19].

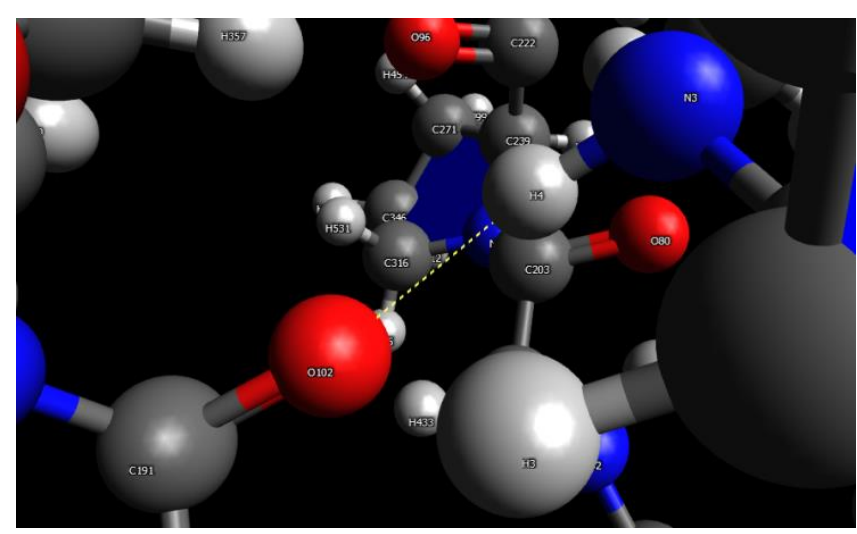

Figure 5. $\mathrm{N}-\mathrm{H}_{(\mathrm{Gly})} \cdots \mathrm{O}=\mathrm{C}_{(\mathrm{Pro})}$ hydrogen bond can exist even without hydrated environment.
The main difference between CLPs and CLPWs models in this study is whether the models include water molecules around them. As showed in Table 2 and 3, the main reason of steric energy differences between CLP and CLPW models is related to VDW and dipole/dipole interaction energies which are directly associated with the existence of water molecules. As expected, the observed hydrogen bonding numbers in CLPW models have higher than that of CLP models (Table 4). Furthermore, the types of hydrogen bonding observed in the models suggest that bound water molecules help in binding collagen triple helix as intrachain and interchain by created water bridges (Figure 6, 7, and 8).

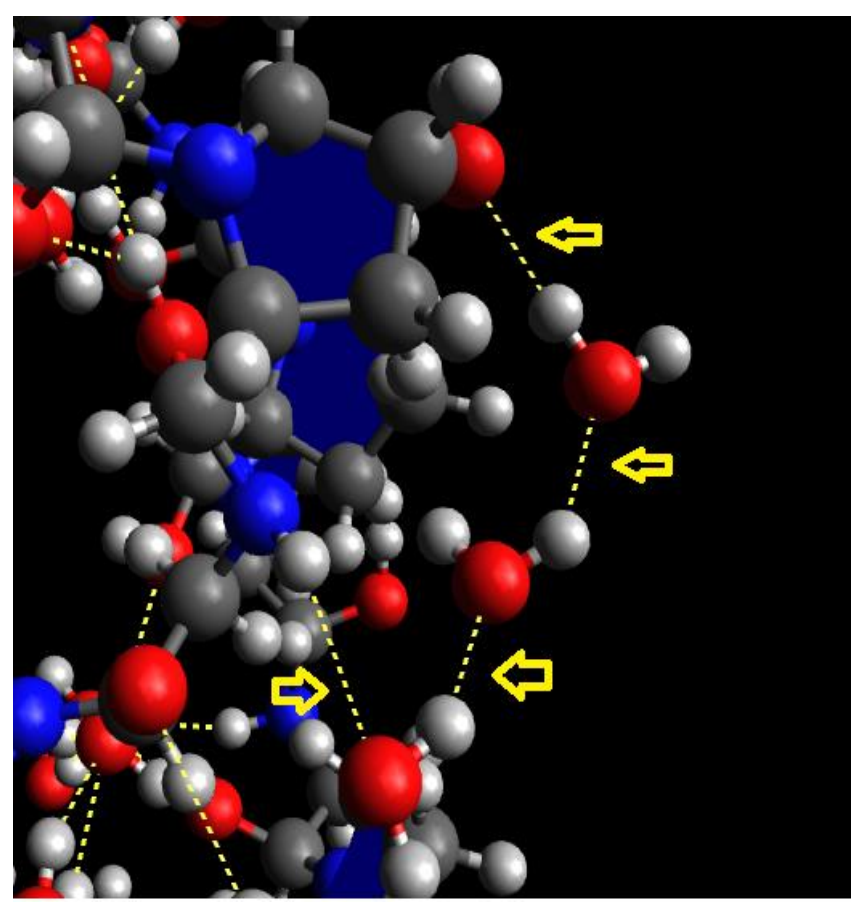

Figure 6. Hydrogen bonding between hydroxyl group of Hyp and N-H group of Gly or Pro through contribution of water bridges ( $\left.\mathrm{N}-\mathrm{H}_{(\mathrm{Gly} \text { or Pro) }} \cdots(\text { Water })_{\mathrm{n}} \cdots \mathrm{O}-\mathrm{H}_{(\mathrm{Hyp})}\right)$.

These results cumulatively suggest that the presence of water inside and around collagen molecule has an important role: increasing the hydrogen bonding sites among collagen chains. Such water molecules create attractive forces on the collagen molecule, resulting in lower energy in collagen. Thus, the structure of collagen becomes more stable with water molecules through providing an extra way for binding triple chains like intrachain and interchain. In the original study by Bella et al [7, 12, 16], they extensively discussed the role of water molecules on the stability of collagen, and showed that water bridges can effectively mediate intrachain and interchain hydrogen bonding. Engel et al. [27] also reported that the stability of both (Pro-Pro-Gly) $)_{n}$ and (ProHyp-Gly $)_{n}$ peptide increase with the existing of water molecules. Confirming with the previously published studies [7, 35-37], this present study also demonstrates the critical role of water mediating hydrogen bonding. 
The second important finding of the present study is related to role of Hyp. The results showed that total steric energy of CLPW1 has lesser than that of CLPW2 and the value has lesser than CLP2 for CLP1. Although the only difference between CLP1 and CLP2 was to replacing Hyp residue with Pro, this small modification in the model leads to a dramatic change in steric energies (Table 2). The hydrogen bond numbers of CLP1 and CLPW1 are much higher than those of CLP2 and CLPW2 (Table 4). Furthermore, some specific water bridges involve directly the presence of Hyp residue and they only exist with the presence of Hyp such as: O$\mathrm{H}_{(\mathrm{Hyp})} \cdots(\text { Water })_{n} \cdots \mathrm{N}-\mathrm{H}_{\text {(Gly or Pro) }}$ and $\mathrm{O}=\mathrm{C}_{(\mathrm{Gly} \text { or Pro) }} \cdots$ $(\text { Water })_{\mathrm{n}} \cdots \mathrm{O}-\mathrm{H}_{\text {(Hyp) }}$ (Figure 7 and 8 ).

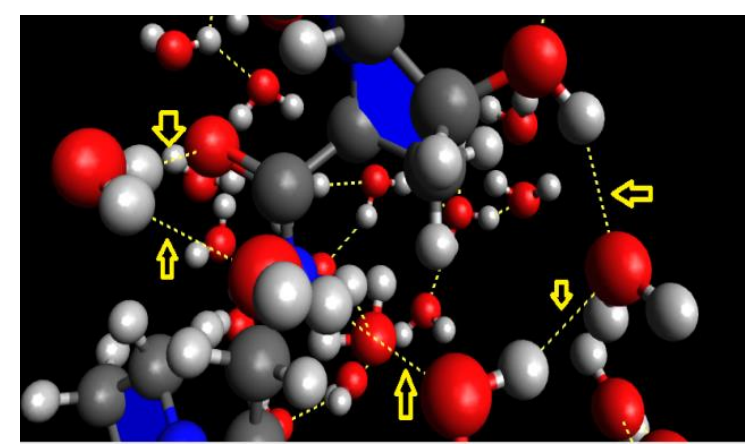

Figure 7. Hydrogen bonding between hydroxyl group of Hyp and carbonyl group of any residues through contribution of water bridges $\left(\mathrm{O}-\mathrm{H}_{(\mathrm{Hyp})} \cdots(\text { Water })_{\mathrm{n}} \cdots \mathrm{O}=\mathrm{C}_{(\mathrm{Gly} \text { or Pro })}\right)$.

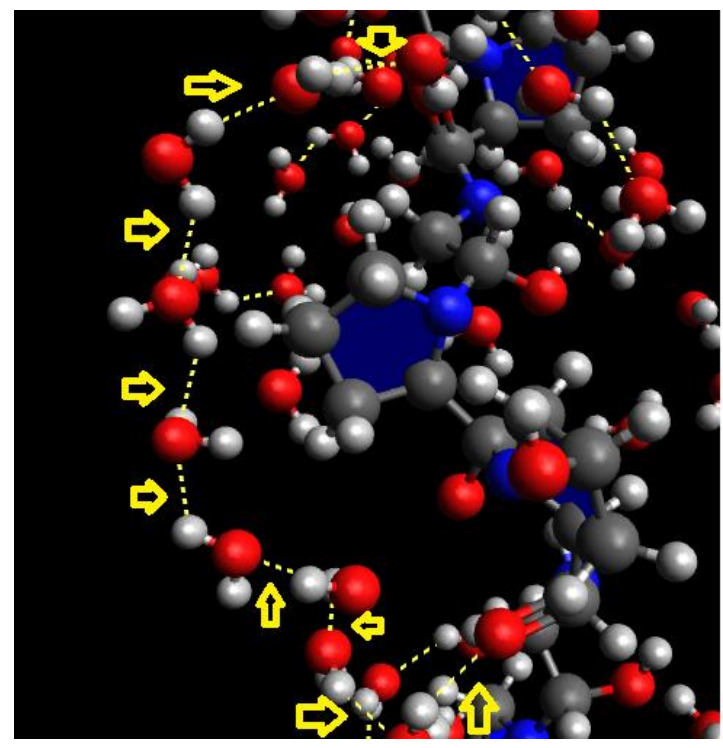

Figure 8: Hydrogen bonding between two carbonyl groups of any residues through contribution of water bridges $\left(\mathrm{C}=\mathrm{O}_{(\text {any residue })} \cdots(\text { Water })_{\mathrm{n}} \cdots \mathrm{O}=\mathrm{C}_{(\text {any residue })}\right)$.

All these results indicated the essential role of Hyp for the collagen molecular stabilization. Previously, Kawahara et al. [38] showed that (Pro-Hyp-Gly) neptide is more stable than (Pro-Pro-Gly) $)_{n}$,suggesting that Hyp residue provides an increase in stability of collagen structure. Furthermore, the studies of Bella et al. [7, 12, 16] showed that Hyp residues mostly interact with surrounding water molecules of collagen and this situation provides more hydrogen bonding with water molecules: thereby, significantly enhancing stability of collagen. Using steric energy concept, this work also confirms this important role of Hyp in collagen stabilization mechanism.

\section{CONCLUSSION}

Using steric energy concept, the overall results suggest that Hyp residue and water molecules around the collagen significantly contribute to the stability of collagen structure with providing extra way to bind each collagen chains each other through several types of water bridges. Such water bridges around the collagen molecule are cumulatively created more attractive forces which consequently decrease the potential energy of collagen molecule, leading to more stable collagen molecule. Previously, the importance of water on mechanical behavior of single collagen molecule has been reported computationally [39-42]. Since uncoiling of collagen type $\mathrm{I}$ is one of the toughening mechanisms observed in bone, the collagen stability could be thought as another essential determinant of bone's resistance to fracture. But, to date, there is no study available showing such association between collagen stability and bone fracture resistance. Therefore, future studies are necessary to investigate the direct relationship between collagen stability and mechanical properties of bone, and the role of Hyp and bound water in this relationship.

\section{ACKNOWLEDGEMENTS}

The author would like to thank Prof. Dr. Ozan Akkus at Case Western Reserve University for providing valuable feedback and suggestions during conceiving the study and drafting the manuscript.

\section{REFERENCES}

[1] M. Unal, A. Creecy, and J. S. Nyman, "The Role of Matrix Composition in the Mechanical Behavior of Bone," Curr Osteoporos Rep, vol. 16, pp. 205-215, June 012018.

[2] L. Knott and A. J. Bailey, "Collagen cross-links in mineralizing tissues: a review of their chemistry, function, and clinical relevance," Bone, vol. 22, pp. 181-187, 1998.

[3] J.-Y. Rho, L. Kuhn-Spearing, and P. Zioupos, "Mechanical properties and the hierarchical structure of bone," Medical Engineering \& Physics, vol. 20, pp. 92-102, 1998.

[4] M. Unal, H. Jung, and O. Akkus, "Novel Raman Spectroscopic Biomarkers Indicate That Postyield Damage Denatures Bone's Collagen," Journal of Bone and Mineral Research, vol. 31, pp. 1015-1025, 2016.

[5] G. N. Ramachandran and G. Kartha, "Structure of collagen," Nature, vol. 176, pp. 593-5, Sep 241955.

[6] A. Rich and F. H. Crick, "The molecular structure of collagen," J Mol Biol, vol. 3, pp. 483-506, Oct 1961.

[7] J. Bella, "Collagen structure: new tricks from a very old dog," Biochemical Journal, vol. 473, pp. 1001-1025, 2016. 
[8] M. D. Shoulders and R. T. Raines, "Collagen Structure and Stability," Annual Review of Biochemistry, vol. 78, pp. 929-958, 2009.

[9] C. L. Jenkins and R. T. Raines, "Insights on the conformational stability of collagen," Natural Product Reports, vol. 19, pp. 49-59, 2002.

[10] G. N. Ramachandran and G. Kartha, "Structure of collagen," Nature, vol. 174, pp. 269-70, Aug 71954.

[11] K. Okuyama, K. Okuyama, S. Arnott, M. Takayanagi, and M. Kakudo, "Crystal and molecular structure of a collagen-like polypeptide (Pro-Pro-Gly) 10," J Mol Biol, vol. 152, pp. 427-443, 1981.

[12] J. Bella, M. Eaton, B. Brodsky, and H. M. Berman, "Crystal and molecular structure of a collagen-like peptide at 1.9 A resolution," Science, vol. 266, pp. 75-81, 1994.

[13] S. Sakakibara, Y. Kishida, K. Okuyama, N. Tanaka, T. Ashida, and M. Kakudo, "Single crystals of (Pro-Pro-Gly) 10, a synthetic polypeptide model of collagen," J Mol Biol, vol. 65 , pp. 371-373, 1972.

[14] E. Suzuki, R. Fraser, and T. MacRae, "Role of hydroxyproline in the stabilization of the collagen molecule via water molecules," International Journal of Biological Macromolecules, vol. 2, pp. 54-56, 1980.

[15] T. V. Burjanadze, "Thermodynamic substantiation of water-bridged collagen structure," Biopolymers: Original Research on Biomolecules, vol. 32, pp. 941-949, 1992.

[16] J. Bella, B. Brodsky, and H. M. Berman, "Hydration structure of a collagen peptide," Structure, vol. 3, pp. 893906, 1995.

[17] N. K. Shah, J. A. Ramshaw, A. Kirkpatrick, C. Shah, and B. Brodsky, "A host- guest set of triple-helical peptides: stability of Gly-XY triplets containing common nonpolar residues," Biochemistry, vol. 35, pp. 10262-10268, 1996.

[18] R. Berisio, L. Vitagliano, L. Mazzarella, and A. Zagari, "Crystal structure determination of the collagen-like polypeptide with repeating sequence Pro-Gyp-Gly: Implications for hydration," Biopolymers, vol. 56, pp. 8-13, 2001.

[19] A. Tamilselvan and D. Zhang, "Effect of hydration on molecular stability of bone collagen," Proceedings of the Ieee 28th Annual Northeast Bioengineering Conference, pp. 197-198, 2002.

[20] K. Mizuno, T. Hayashi, D. H. Peyton, and H. P. Bächinger, "Hydroxylation-induced Stabilization of the Collagen Triple Helix ACETYL-(GLYCYL-4 (R)HYDROXYPROLYL-4 (R)-HYDROXYPROLYL) 10NH2 FORMS A HIGHLY STABLE TRIPLE HELIX," Journal of Biological Chemistry, vol. 279, pp. 38072-38078, 2004.

[21] J. A. Howard, V. J. Hoy, D. O'Hagan, and G. T. Smith, "How good is fluorine as a hydrogen bond acceptor?," Tetrahedron, vol. 52, pp. 12613-12622, 1996.

[22] S. K. Holmgren, K. M. Taylor, L. E. Bretscher, and R. T. Raines, "Code for collagen's stability deciphered," Nature, vol. 392, p. 666, 1998.

[23] S. K. Holmgren, L. E. Bretscher, K. M. Taylor, and R. T. Raines, "A hyperstable collagen mimic," Chemistry \& biology, vol. 6, pp. 63-70, 1999.
[24] T. Hiyama, K. Kanie, T. Kusumoto, Y. Morizawa, and M. Shimizu, "Properties of fluoroorganic compounds," Organofluorine Compounds: Chemistry and Applications, pp. 10-12, 2000.

[25] Y. Nishi, S. Uchiyama, M. Doi, Y. Nishiuchi, T. Nakazawa, T. Ohkubo, and Y. Kobayashi, "Different effects of 4-hydroxyproline and 4-fluoroproline on the stability of collagen triple helix," Biochemistry, vol. 44, pp. 6034-6042, 2005.

[26] R. W. Newberry and R. T. Raines, "4Fluoroprolines: Conformational analysis and effects on the stability and folding of peptides and proteins," in Peptidomimetics I, ed: Springer, 2016, pp. 1-25.

[27] J. Engel, H. T. Chen, D. J. Prockop, and H. Klump, "The triple helix $\rightleftharpoons$ coil conversion of collagen-like polytripeptides in aqueous and nonaqueous solvents. Comparison of the thermodynamic parameters and the binding of water to (L-Pro-L-Pro-Gly) $n$ and (L-Pro-L-HypGly) n," Biopolymers: Original Research on Biomolecules, vol. 16, pp. 601-622, 1977.

[28] D. L. Bodian, R. J. Radmer, S. Holbert, and T. E. Klein, "Molecular dynamics simulations of the full triple helical region of collagen type I provide an atomic scale view of the protein's regional heterogeneity," in Biocomputing 2011, ed: World Scientific, 2011, pp. 193-204.

[29] T. A. Halgren, "Merck molecular force field. I. Basis, form, scope, parameterization, and performance of MMFF94," Journal of computational chemistry, vol. 17, pp. 490-519, 1996.

[30] U. Burkert, "Molecular mechanics," ACS monograph 177, 1982.

[31] T. W. Shattuck, "Colby College Molecular Mechanics Tutorial," ed: Colby College, Waterville, 2008.

[32] M. D. Hanwell, D. E. Curtis, D. C. Lonie, T. Vandermeersch, E. Zurek, and G. R. Hutchison, "Avogadro: an advanced semantic chemical editor, visualization, and analysis platform," Journal of cheminformatics, vol. 4, pp. 117, 2012.

[33] R. Fraser, T. MacRae, and E. Suzuki, "Chain conformation in the collagen molecule," J Mol Biol, vol. 129, pp. 463-481, 1979.

[34] J. K. Rainey and M. C. Goh, "A statistically derived parameterization for the collagen triple-helix," Protein Science, vol. 11, pp. 2748-2754, 2002.

[35] I. Streeter and N. H. de Leeuw, "A molecular dynamics study of the interprotein interactions in collagen fibrils," Soft matter, vol. 7, pp. 3373-3382, 2011.

[36] I. Fu, D. A. Case, and J. Baum, "Dynamic watermediated hydrogen bonding in a collagen model peptide," Biochemistry, vol. 54, pp. 6029-6037, 2015.

[37] M. C. Tourell and K. I. Momot, "Molecular dynamics of a hydrated collagen peptide: insights into rotational motion and residence times of single-water bridges in collagen," The Journal of Physical Chemistry B, vol. 120, pp. 12432-12443, 2016.

[38] K. Kawahara, Y. Nishi, S. Nakamura, S. Uchiyama, Y. Nishiuchi, T. Nakazawa, T. Ohkubo, and Y. Kobayashi, "Effect of hydration on the stability of the collagen-like triple-helical structure of [4 (R)-hydroxyprolyl-4 (R)- 
hydroxyprolylglycine] 10," Biochemistry, vol. 44, pp. 15812-15822, 2005.

[39] D. Zhang, U. Chippada, and K. Jordan, "Effect of the structural water on the mechanical properties of collagenlike microfibrils: a molecular dynamics study," Ann Biomed Eng, vol. 35, pp. 1216-1230, 2007.

[40] A. Gautieri, S. Vesentini, A. Redaelli, and M. J. Buehler, "Intermolecular slip mechanism in tropocollagen nanofibrils," International Journal of Materials Research, vol. 100, pp. 921-925, 2009.

[41] A. Gautieri, M. I. Pate, S. Vesentini, A. Redaelli, and M. J. Buehler, "Hydration and distance dependence of intermolecular shearing between collagen molecules in a model microfibril," J Biomech, vol. 45, pp. 2079-2083, 2012.

[42] H. Ghodsi and K. Darvish, "Investigation of mechanisms of viscoelastic behavior of collagen molecule," J Mech Behav Biomed Mater, vol. 51, pp. 194-204, 2015. 